\title{
Erratum to: Navigated MRI-guided liver biopsies in a closed-bore scanner: experience in 52 patients
}

\author{
Michael Moche $^{1} \cdot$ Susann Heinig $^{1} \cdot$ Nikita Garnov $^{1} \cdot$ Jochen Fuchs $^{1}$. \\ Tim-Ole Petersen ${ }^{1}$ - Daniel Seider ${ }^{1} \cdot$ Philipp Brandmaier $^{1}$ - Thomas Kahn ${ }^{1}$. \\ Harald Busse ${ }^{1}$
}

Published online: 17 December 2015

(C) European Society of Radiology 2015

\section{Erratum to: European Radiology}

\section{DOI 10.1007/s00330-015-4097-1}

Due to a multifactorial transcription error, Philipp Brandmaier, M.D., from the Department of Diagnostic and Interventional Radiology, Leipzig University Hospital, was inadvertently excluded from the author list in the original publication; this has since been corrected.

We apologise for this error.

The online version of the original article can be found at http://dx.doi.org/ 10.1007/s00330-015-4097-1.

Michael Moche

Michael.Moche@medizin.uni-leipzig.de

1 Department of Diagnostic and Interventional Radiology, Leipzig University Hospital, Liebigstr. 20, 04103 Leipzig, Germany 